\title{
Toucher la pollution industrielle du doigt grâce aux lichens
}

Ethnographie d'une observation scientifique et citoyenne de l'environnement à Fos-sur-Mer

Christelle Gramaglia et Charles-Enzo Dauphin

\section{(2) OpenEdition}

Journals

Édition électronique

URL : https://journals.openedition.org/tc/8610

DOI : $10.4000 /$ tc. 8610

ISSN : 1952-420X

Éditeur

Éditions de l'EHESS

Référence électronique

Christelle Gramaglia et Charles-Enzo Dauphin, « Toucher la pollution industrielle du doigt grâce aux lichens », Techniques \& Culture [En ligne], Suppléments aux numéros, mis en ligne le 18 décembre 2017, consulté le 01 octobre 2022. URL : http://journals.openedition.org/tc/8610 ; DOI : https:// doi.org/10.4000/tc. 8610

Ce document a été généré automatiquement le 1 octobre 2022.

Tous droits réservés 


\section{Toucher la pollution industrielle du doigt grâce aux lichens}

Ethnographie d'une observation scientifique et citoyenne de l'environnement à Fos-sur-Mer

\section{Christelle Gramaglia et Charles-Enzo Dauphin}

Nous tenons à remercier Élisabeth Cartoux, responsable du département de botanique du Muséum Henri-Lecoq de Clermont-Ferrand, auteure d'une courte mais précieuse biographie de William Nylander, qui nous a reçus et accordé de son temps.

1. La ZIP de Fos vue de la vieille ville de Fos-sur-Mer

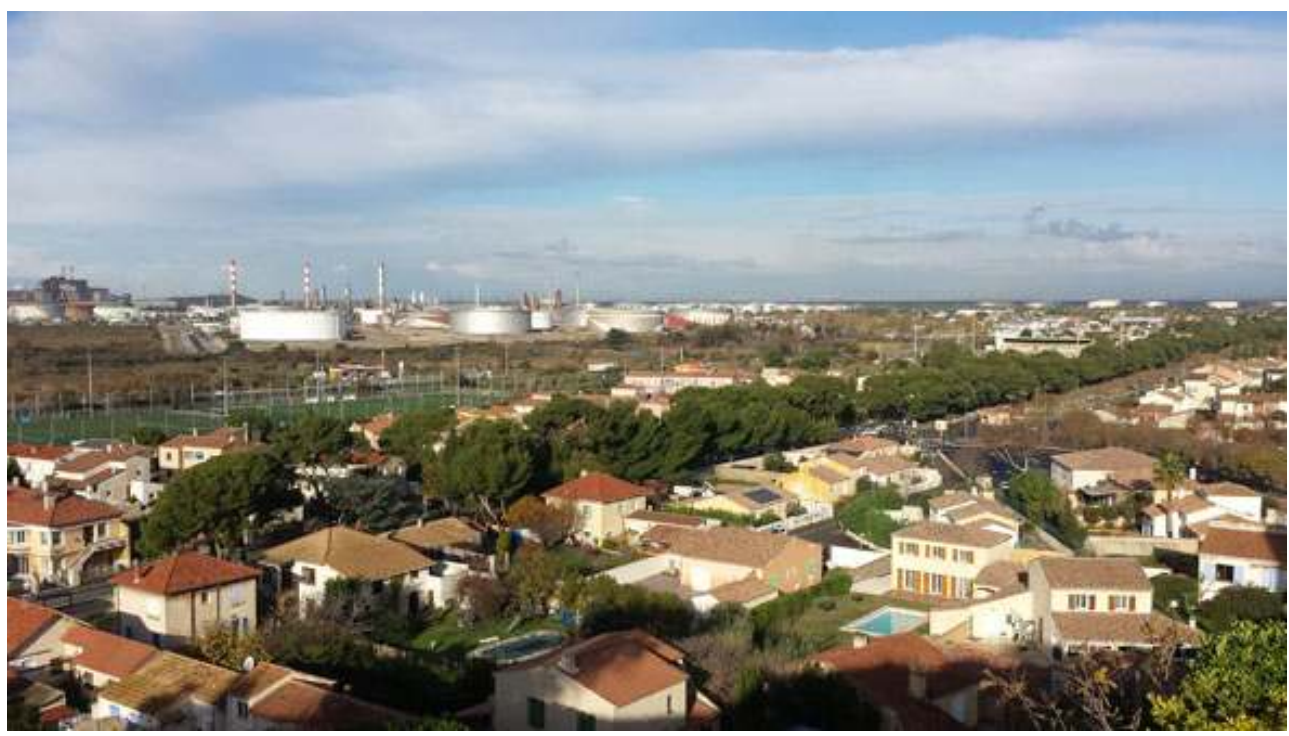

C. Gramaglia

La zone industrialo-portuaire (ZIP) de Fos ${ }^{1}, 10000$ ha environ, est l'une des plus grandes d'Europe (Photo 1). Elle a été construite à la fin des années 1960 sur le littoral provençal, en lieu et place de la steppe de la Crau, des prairies, des marais et des étangs cernant les villes de Fos-sur-Mer et Port-Saint-Louis du Rhône. En une décennie, des 
usines métallurgiques et pétrochimiques s'y sont installées, en même temps que des darses étaient creusées pour accueillir tankers et porte-containers. Les bouleversements sociaux et territoriaux ont été majeurs (Paillard 1981, Daumalin \& Gramaglia à paraître). Presque immédiatement, la pollution a généré des craintes et des protestations, notamment parce que les autorités avaient prévu de déclarer la zone insalubre pour limiter l'urbanisation, sans tenir compte des populations qui y habitaient déjà. Dès 1971, une mission du Ministère de l'environnement était toutefois diligentée pour remédier à la situation de plus en plus conflictuelle. Elle déboucha sur la création du premier Secrétariat permanent pour les problèmes de pollution industrielle (S3PI), pour renforcer la coordination entre services de l'État, d'un côté, et entre industriels, d'un autre. Des normes de qualité de l'air et de l'eau furent ensuite définies, a posteriori, avec l'Association des industriels de Fos-étang de Berre pour l'étude et la prévention de la pollution (AIRFOBEP), fondée en 1972. Des solutions techniques furent également mises en place pour réduire émissions et effluents industriels.

1 Les préoccupations environnementales et sanitaires des riverains ont dès lors fluctué, notamment sous l'effet de la crise économique de la fin des années 1970, qui a fait de l'emploi leur premier souci. Il a fallu attendre la relance industrielle des années 2000, la construction d'un incinérateur, de nouvelles installations gazières et logistiques, pour que de nouveau, elles s'expriment. Sous la pression de mobilisations particulièrement intenses (Girard 2012, Osadtchy 2015), les élus de la communauté de communes Ouest Provence ont alors demandé un état des lieux environnemental du territoire, avant de soutenir la création d'un organisme scientifique indépendant qui se consacre entièrement à ces problèmes, l'Institut écocitoyen pour la connaissance des pollutions (IECP), en 2010. Cette association loi 1901, implantée au cœur de la ZIP, développe des recherches participatives originales sur les effets de l'accumulation de substances chimiques sur l'environnement et la santé humaine. Elle cible l'ensemble des milieux et des organismes vivants, exerçant à la fois une mission de lanceur d'alerte (Chateauraynaud \& Torny 2005) et de sentinelle (Gramaglia \& Sampaio da Silva 2011), capable d'identifier des problèmes que les instruments, les normes et les seuils réglementaires ne permettent pas toujours de cerner.

2 Notre article traite d'une expérience de biosurveillance scientifique et citoyenne lancée ces dernières années sur le territoire de la ZIP par l'IECP. Il montre comment un réseau d'acteurs locaux compétents s'est mis en place pour penser et connaitre avec les lichens, des êtres symbiotiques de champignons et d'algues, particulièrement petits voire insignifiants puisqu'ils ne font pas l'objet d'une attention particulière ou d'un usage commun sous nos latitudes ${ }^{2}$, mais permettent néanmoins d'accéder aux mondes infimes des phénomènes toxiques et de "toucher du doigt la pollution». Suivant les pistes de réflexion ouvertes par la sociologie des sciences, notamment des travaux sur les sciences citoyennes (Irwin 1995, Charvolin, Micoud \& Nyhart 2007), l'extension des collectifs de recherche (Callon, Lascoumes \& Barthe 2001) et les ignorances dans le domaine de l'évaluation des risques (Lasch, Sersynznski \& Wynne 1996, Frickel \& Edwards 2013), nous aborderons les critiques adressées à la surveillance réglementaire de l'environnement (Ottinger 2010, Kinchy, Parks \& Jalbert 2015). Avec une approche très influencée par la théorie de l'acteur-réseau (Callon 1986, Clarke \& Fujimura 1996, Latour 2001), nous nous demanderons aussi ce que des associations inédites d'humains et non-humains, ici qualifiés d'agencements multispécifiques eu égard à leurs 
potentialités (Kirksey \& Helmreich 2010), changent à la façon de produire des savoirs. Quels sont les bénéfices scientifiques et sociaux de ces méthodes par comparaison avec les possibilités de quantification croissantes offertes par les instruments de mesure de laboratoire ? Nous montrerons que la contre-surveillance ainsi exercée « avec » (Tsing 2012, de la Bellacasa 2012) les lichens et à partir de la perspective de petits êtres littéralement attachés à leur environnement, induit une forme de "compagnonnage " (Haraway 2008) qui transforme non seulement les connaissances sur la pollution, mais aussi le regard porté sur le territoire, obligeant ceux qui s'y adonnent, scientifiques et citoyens, à réfléchir « ensemble » aux conditions de son habitabilité (Blanc 2010).

\section{Les critiques de la surveillance de l'environnement}

La surveillance réglementaire de la qualité de l'air dans les villes proches de la ZIP de Fos est assurée par l'association agréée AIRPACA, créée en 2012, après la fusion d'AIRFOPEB et ATMO PACA, deux associations agréées pour la surveillance de la qualité de l'air (AASQA). La nouvelle entité dispose de trois stations permanentes équipées en appareils de mesure en zone urbaine ou périurbaine, une à Port-Saint-Louis du Rhône et deux à Fos-sur-Mer. Trois autres sont installées à Port-de-Bouc, dont une spécifiquement en zone industrielle. Le suivi, normé sur le plan européen, est focalisé sur des polluants précis, dont le dioxyde de soufre, l'oxyde d'azote, les particules fines (en anglais PM - Particulate Matter - 10), des métaux (arsenic, nickel), des hydrocarbures (benzène) et l'ozone. AIRPACA dispose par ailleurs d'un laboratoire mobile. L'association peut, selon les besoins, lancer des études ponctuelles sur d'autres polluants que ceux surveillés en routine (par exemple, mercure, pesticides ou encore particules très fines, PM 2,5). Par ailleurs, elle recourt à la modélisation pour réaliser des prévisions et informer les populations d'éventuels pics de pollution ${ }^{3}$.

3 Même si la qualité générale de l'air de la ZIP et de ses environs s'est globalement améliorée depuis ces vingt dernières années, de nombreux problèmes subsistent, avec des dépassements des seuils réglementaires assez réguliers qui viennent s'ajouter à des nuisances, notamment olfactives. Dans sa synthèse la plus récente, AIRPACA relève ainsi des niveaux de pollution en hausse en 2015 à Fos-sur-Mer et Port-Saint-Louis du Rhône, par rapport à 2013 et 2014, «en raison de conditions météorologiques moins favorables à la dispersion des polluants " ${ }^{4}$. Quel que soit le nombre des mesures réalisées dans le cadre de la surveillance réglementaire, elles n'ont pas permis de calmer les inquiétudes des populations riveraines de la ZIP de Fos qui se demandaient, en 2006 et 2007, si elles n'étaient pas exposées à des risques excessifs dans une pétition envoyée à la Commission des pétitions du Parlement européen ${ }^{5}$. Des doutes sont en effet régulièrement exprimés sur la capacité des méthodes et des instruments usuels à saisir la complexité des phénomènes en cause, c'est-à-dire l'accumulation et les effets de synergie des faibles doses sur le temps long. Ces critiques observées à Fos-sur-Mer font écho à d'autres protestations dont la littérature rend compte à propos d'autres sites ou fronts industriels sacrifiés, pour reprendre l'expression du journaliste nordaméricain Steven Lerner (2010). Celles-ci sont de plusieurs ordres. Des recherches récentes en sociologie des sciences ont ainsi montré que les évaluations des risques avaient tendance à reproduire les ignorances (Frickel, Edwards 2013), en concentrant à la fois spatialement et épistémologiquement les ressources disponibles sur un nombre réduit de lieux et de substances à suivre, et en recourant abusivement à l'extrapolation des données sur la contamination (à d'autres lieux où les mesures ne sont pas faites et à 
d'autres substances pour lesquelles les valeurs toxicologiques ne reposent pas sur des connaissances établies, par exemple). Des ignorances peuvent être intentionnellement et même frauduleusement entretenues. Plus nombreuses seraient encore, selon les auteurs, les lacunes résultant des habitudes, des méthodes, des instruments et des normes en vigueur dans un domaine ou un groupe professionnel d'experts orientent sans l'expliciter, le processus de production des connaissances. Ainsi, des pans entiers de recherche seraient laissés sans financement, dans un état d'incomplétude potentiellement préjudiciable aux populations concernées, notamment pour ce qui concerne les études sur les effets des pollutions sur l'environnement et la santé dans les territoires industrialisés (Frickel et al. 2010).

Pour combler les ignorances, de nombreuses initiatives citoyennes soutenues par les mouvements pour la justice environnementale nord-américains ont été lancées à partir des années 1990, dans le sillage des mobilisations de profanes sur des questions environnementales et sanitaires (Akrich, Barthe \& Rémy 2013). Elles visent à inciter les riverains à produire leurs propres données en recourant à des instruments bricolés peu onéreux comme des seaux d'échantillonnage de l'air. L'objectif est de former de véritables «brigades " (Ottinger 2009), capables d'assurer une présence continue sur le terrain et de repérer des anomalies, par exemple, des pics de pollution auxquels les organismes officiels de surveillance de la qualité de l'air ne prêtent pas attention parce qu'ils raisonnent surtout à partir de moyennes mensuelles ou annuelles, conformément à la réglementation. Les prélèvements de ces «brigades » sont analysés en laboratoire, puis les résultats présentés à la fois aux autorités, notamment l'Environnemental Protection Agency (EPA), et aux industriels sommés de rendre des comptes. Cette production de données nouvelles ou contradictoires s'est avérée fort utile. Des recherches en sciences humaines et sociales ont ainsi montré que ces mobilisations avaient pu induire des changements (Ottinger 2010a, 2010b). Cela, bien que les militants aient rencontré des difficultés pour faire reconnaître leurs compétences et leur légitimité dans un domaine, la surveillance environnementale, très largement accaparé par des experts scientifiques et techniques ${ }^{6}$.

5 En réponse aux objections couramment avancées, mettant en cause la validité des mesures citoyennes réalisées avec les seaux, des Organisations Non Gouvernementales ont alors entrepris de doter les riverains concernés d'appareils plus sophistiqués et surtout normalisés, soit des capteurs pour les pollutions atmosphériques, soit des préleveurs automatiques pour les pollutions aquatiques, notamment pour surveiller les impacts environnementaux de l'exploitation des gaz de schiste qui échappe à la loi sur l'eau américaine (Jalbert 2016). Ce recourt à la technologie, s'il permet de clore les controverses sur la part de subjectivité des observations profanes, n'est pas sans poser problème. D'abord, il tend à renforcer une division du travail qui est souvent pointée du doigt dans les expériences de science citoyenne, les riverains étant dépendants de tiers pour la maintenance des instruments, mais aussi l'interprétation de gros volumes de données ${ }^{7}$. Ensuite, il tend à déposséder ces mêmes riverains de ce qui faisait leur force, c'est-à-dire leurs savoirs expérientiels et contextuels, leur capacité à subvertir les protocoles et métrologies standards et à soulever des questions autrement négligées (Jalbert \& Kinchy 2016). Il peut également générer des tensions fortes au sein des groupes de citoyens mobilisés, et entre eux, selon qu'ils ont accès (ou pas) à des instruments onéreux, et qu'ils valorisent (ou pas) les données que ces derniers permettent d'acquérir de manière mécanique et automatisée. 
Comme en matière de participation politique, le bilan est donc mitigé (Blondiaux \& Fourniau 2011). On peut se féliciter que les collectifs de recherche s'élargissent (Callon, Lascoumes \& Barthe 2001), en grande partie suite à des protestations citoyennes permettant de faire reconnaître d'autres formes d'expertise, mais il faut reconnaître que les objectifs de la démocratie technique sont loin d'être atteints. Il reste compliqué pour les riverains affectés par des pollutions industrielles d'obtenir des autorités qu'elles leur fournissent les données qu'elles possèdent, et plus encore, d'influer sur la production de connaissances nouvelles et les décisions qui peuvent en découler. La façon dont on définit les valeurs toxicologiques de référence, les normes et les seuils, mais également les méthodes d'évaluation des risques, n'a pas vraiment changé. Elle reste entre les mains des seuls experts des domaines concernés, et sous l'influence des industriels (Jouzel \& Lascoumes 2011), ce qui complique l'exercice d'une vigilance citoyenne, et plus encore la possibilité de promouvoir une science "par le bas" (Harding 2008).

\section{Un réseau de volontaires pour l'observation de l'environnement}

L'IECP a été créé en 2010, suite à des pressions des associations locales engagées contre la construction de l'incinérateur de la Communauté urbaine de Marseille dans la ZIP de Fos (Osadtchy 2016). Celles-ci avaient exigé un bilan environnemental avant le démarrage de nouvelles activités industrielles. L'étude qui y fut consacrée, Approche intégrée de la gestion des risques environnementaux à l'échelle d'un territoire (AIGRETTE), conduite par le Bureau de recherches géologiques et minières (BRGM) en concertation avec différentes catégories d'acteurs du territoire, a permis d'identifier des points noirs où s'accumule la pollution, mais aussi de nombreuses ignorances relatives à la qualité de l'eau, de l'air et des sols du territoire (Blanchard et al. 2009). Le rapport lui-même a fait l'objet de vives discussions au moment de sa restitution lors de réunions publiques, tant pour le choix des méthodes, que des lieux de prélèvement, ou le choix de ne pas traiter des questions épidémiologiques. Cela nous a été répété maintes fois au cours de notre enquête de terrain ${ }^{8}$. Dans ce climat de suspicion et de défiance, les élus de la communauté de communes Ouest-Provence ont alors pris l'engagement de poursuivre les recherches en dotant le territoire d'un outil de connaissance indépendant, capable de scruter l'état de santé des riverains de la ZIP et de leur environnement sur le temps long. Ils ont décidé de consacrer une partie des recettes fiscales générées par le site de traitement de déchets qu'ils n'ont pu empêcher, au financement d'une association scientifique de type loi 1901, soit environ un demi-million d'euros. Après plusieurs mois de préparation, les premières études sur les sources d'émissions atmosphériques, les particules fines et la bioimprégnation des lichens ont pu être lancées, en 2011. Une équipe de permanents à dominante scientifique a été embauchée pour ce faire, un directeur (ingénieur chimiste), un responsable scientifique (docteur en chimie de l'environnement), une spécialiste de questions de santé (docteure en chimie et santé), un technicien, une responsable administrative et une professionnelle de la communication, de même qu'un doctorant en sciences de l'environnement de l'Université de Marseille, recruté spécialement pour mener des recherches sur les particules fines (PM 2,5). D'autres scientifiques les ont depuis rejoints pour apporter des compétences complémentaires, par exemple, une écotoxicologue (docteur en 
physiologie et génétique moléculaire) et un chargé de mission (ingénieur en écologie et biosciences). On compte désormais aussi un gestionnaire et un responsable des finances.

7 L'originalité de l'IECP tient à sa structuration. Outre son personnel scientifique, il s'appuie sur un réseau des Volontaires pour l'observation citoyenne de l'environnement (VOCE). Celui-ci est chargé à la fois de faire remonter des témoignages et des questions à investiguer, et de la mise en œuvre de certaines actions de recherche. L'implication des volontaires est favorisée par la valorisation de leurs savoirs et savoirfaire à différents stades, mais aussi grâce à des formations adaptées qui peuvent les aider à formuler des questions, étayer leurs arguments et réunir des preuves sur des problèmes qu'ils pourraient suspecter ou avoir besoin de documenter. L'objectif est de produire des connaissances qui soient pertinentes pour le territoire, au sens où elles répondent à des interrogations des riverains, comblent des ignorances, tout en traitant des spécificités de la ZIP, c'est-à-dire des effets du cumul de très nombreuses émissions et effluents industriels. Il est également politique, puisqu'il s'agit de leur donner accès, ainsi qu'aux collectivités locales qui les représentent, à des connaissances nouvelles et des preuves susceptibles de renforcer leur capacité d'argumentation, mais aussi d'action.

8 Le réseau VOCE compte aujourd'hui une cinquantaine de volontaires, résidant dans plusieurs localités du golfe de Fos: Fos-sur-Mer pour le plus grand nombre, mais également Port-Saint-Louis-du-Rhône, Port-de-Bouc, Martigues, Lavéra et Istres, pour les autres (un réseau complémentaire a également été constitué dans un site témoin, Grans, éloigné des émissions industrielles). Leur contribution à l'effort de recherche collectif se fait selon leurs aptitudes, motivations et préférences, l'observation citoyenne prolongeant souvent une pratique amateur devenue passion cognitive (Gramaglia 2009, Charvolin, Roux \& Demain 2013). C'est le cas de certains pêcheurs ou sportifs qui ont une bonne connaissance du territoire, sont les témoins des changements qui l'affectent, et possèdent aussi du matériel et des compétences techniques. Ainsi Olivier, retraité et membre l'association des plaisanciers de Fos-surMer, proche de l'Association de défense et de protection du littoral du golfe de Fos (ADPLGF), a décidé de prendre part aux recherches menées en mer, avant tout pour partager ses connaissances :

"Je suivais les réunions, on se voyait et puis, ils m'ont demandé si je ne voulais pas participer. Il y a quelques années, 4 ou 5 ans de ça. Il y avait une enquête sur la mer, l'environnement... Donc, comme j'ai le bateau... Ça fait quand même 40 ans, oui, 40 ans qu'on est sur le golfe de Fos, et je connais un peu ses particularités. On a toujours eu un bateau, et je fais de la plongée... ».

On retrouve une motivation comparable, chez Didier, ouvrier de la pétrochimie, plaisancier, ornithologue et botaniste amateur. Propriétaire d'un bateau, il s'est également porté volontaire pour la surveillance du milieu marin. Il en a profité pour partager ses inquiétudes relatives à la raréfaction des poissons dans des secteurs de la ZIP où de nouvelles installations industrielles ont été implantées, même s'il ne pêche pas souvent:

"À l'origine, ils cherchaient des bénévoles pour faire des relevés sur la nature des environs pour une enquête. Je trouvais que ça pouvait être intéressant de livrer toutes mes connaissances, tout ce que je savais du coin... C'était plutôt sur le volet marin... Depuis la construction du méthanier, il y a 
eu quand même pas mal de chamboulements. Aujourd'hui, on en subit un peu les effets... Avant il y avait du poisson en abondance, mais aujourd'hui, selon les saisons, il y a quasiment rien... » nautique de Port-de-Bouc, mais aussi l'ADPLGF, pilier des mobilisations des années 2000 contre l'incinérateur et d'autres projets de développement de la ZIP, fournissent les $2 / 3$ $\mathrm{du}$ contingent des VOCE. Se côtoient des amateurs qui veulent rendre service, tantôt par solidarité, tantôt par intérêt naturaliste, et des militants. Comme Jacques, retraité de l'industrie, joggeur et jardinier, qui revendique un engagement social fort et son « amour » pour la nature, qui l'incite à prendre du temps pour « l'observer ». Il ne s'est pas impliqué lors des mobilisations contre l'incinérateur, mais fait partie du Comité de feu auquel il consacre beaucoup de temps. Il s'exclame :

\section{«Si je peux être utile, moi! J'avais planté des salades dans mon jardin [pour l'IECP]... Dès qu'on me parle de pollution... » [il fronce les sourcils, évoque sa sensibilité personnelle, ses difficultés à courir quand le vent du Sud-Ouest rabat les émissions industrielles sur les collines de Fos-sur-Mer].}

11 Pour Hervé, technicien retraité de l'industrie, grand sportif et dirigeant d'un club de loisir (sa compagne a milité à l'ADPLGF), l'intérêt est double: partager ses connaissances de terrain, mais aussi s'informer sur les changements environnementaux.

«Ils nous ont demandés, au départ, et puis c'est vrai que l'avenir du Golfe, c'est intéressant. C'est notre terrain de jeu, on vit avec. C'est bien de voir comment il évolue. Mais au départ, ils nous ont sensibilisés, et puis on a adhéré ".

12

$$
\begin{aligned}
& \text { Le dernier tiers des volontaires rassemble plutôt des personnes curieuses, désireuses } \\
& \text { d'en apprendre plus sur la pollution, mais également de faire « quelque chose pour le } \\
& \text { territoire », compte tenu d'un rapport de force local particulièrement défavorable. } \\
& \text { Parmi nos interviewés, plusieurs nous ont dit qu'ils partageaient les indignations des } \\
& \text { associations comme l'ADPLGF ou encore l'ancien Collectif santé environnement de } \\
& \text { Port-Saint-Louis du Rhône, mais ne s'étaient pas mobilisés contre l'incinérateur, par } \\
& \text { découragement. Par contre, souhaitant aider l'IECP à étudier les effets de l'ensemble } \\
& \text { des activités industrielles de la ZIP sur l'environnement et la santé des populations, ils } \\
& \text { ont répondu favorablement à ses appels. C'est le cas de David, technicien retraité, } \\
& \text { ancien régatier qui s'est formé à l'observation des lichens. Il souhaitait obtenir des } \\
& \text { données permettant de confondre les industriels et, éventuellement, d'exiger des } \\
& \text { améliorations: } \\
& \text { "Que pouvions-nous faire ? Seulement essayer de savoir pour agir... Le but, } \\
& \text { c'est d'identifier la pollution en fonction des usines. Quand il y a du vent, } \\
& \text { mesurer juste au-dessous... On veut savoir qui fait quoi, et pas avoir une } \\
& \text { estimation globale, comme le font d'ordinaire les pouvoirs publics. Alors, si } \\
& \text { on est capable de confronter l'industriel responsable avec les bonnes } \\
& \text { données, disant qu'on a été capable de contrôler la pollution ce jour-là en } \\
& \text { particulier, parce que c'est le seul à rejeter cette substance-là, on peut le } \\
& \text { mettre face à ses responsabilités... Alors, on peut faire quelque chose ». }
\end{aligned}
$$

Les différentes expériences de sciences participatives conduites par l'IECP, qu'il s'agisse des prélèvements d'eau, de poissons, de l'observation des lichens ou encore de la 
culture de pétunias ou de salades, répondent à des objectifs complémentaires, soit produire des données sur les pollutions qui affectent l'air, l'eau et les sols. Les volontaires y voient l'occasion de mieux comprendre la situation dans laquelle ils sont plongés. Peut-être pensent-ils aussi retrouver des prises sur leur environnement et les phénomènes qui peuvent le dégrader. Carole, ingénieure qui apprécie les loisirs nautiques sans les pratiquer intensivement, nous rappelle que la pollution est en partie invisible à Fos-sur-Mer, qu'il est possible d'y vivre sans y penser continuellement (et en appréciant les avantages matériels qu'on y trouve parce que la commune a une politique sociale particulièrement généreuse, d'ailleurs rendue possible par les revenus fiscaux tirés de l'industrie). Sa participation à une campagne de suivi lichénique lui a donné des éléments supplémentaires pour qualifier ses perceptions, parfois contradictoires. L'avantage qu'elle y a vu, c'est de contribuer à produire des informations utiles pour mieux connaitre voire choisir son lieu de vie. Pour des raisons avant tout professionnelles, elle a quitté Fos-sur-Mer pour s'installer à Istres quelques mois après la première campagne d'observation.

«J'ai été très surprise. Finalement, on pouvait voir de très près ce que c'est que la pollution. On pouvait la toucher, si je puis dire... Alors, on commence à comprendre dans quel genre d'endroit on vit... Honnêtement, selon où vous habitez à Fos, quand vous êtes au centre, vous ne voyez pas les usines. Quand vous êtes chez vous, non plus, vous ne les voyez pas ».

Les volontaires conservent une posture modeste. Nous avons pu l'observer à l'occasion de plusieurs réunions plénières où se sont retrouvés tous ceux qui étaient impliqués dans des recherches afférentes à l'état des milieux terrestres, aquatiques ou atmosphériques. Ils font preuve d'une grande déférence à l'égard des scientifiques de l'IECP. Cette attitude contraste avec les doutes, la méfiance et même la défiance qui peuvent s'exprimer à propos des autorités, par exemple, les services de l'État en charge de la surveillance environnementale, et certains industriels accusés de « ne pas jouer le jeu ", c'est-à-dire qu'ils ne mettraient pas les moyens nécessaires à la production de données pertinentes pour les populations, pas plus qu'ils ne recourraient aux meilleures techniques disponibles pour limiter leurs rejets dans l'environnement. Les récits que nous avons recueillis abondent d'anecdotes visant à illustrer ces mauvaises pratiques, et le sentiment de mépris qui en découle pour les riverains qui ont tenté d'en savoir plus. Dans ce contexte, l'IECP qui se présente comme un organisme scientifique indépendant, bien qu'étant ancré dans le territoire (et financé en grande partie par les collectivités locales), apparaît comme une garantie. Les volontaires s'accommodent relativement bien de la division des tâches telle qu'elle a été définie dans les statuts de l'association (fluidifiée par des procédures leur permettant de faire entendre leur voix à différents moments : recueil des propositions d'étude, coconstruction des protocoles, formation, participation à la collecte des données, au suivi et à la discussion des résultats). Ils ne cherchent pas vraiment, comme dans d'autres expériences de recherche citoyenne (Irwin 1995) ou de " plein air» (Callon, Lascoumes \& Barthe 2001) à contester les méthodes, subvertir les protocoles, réorienter les questions et efforts de recherche ou encore à en prendre le leadership. Ils ne se positionnent pas de cette manière à la différence de certains groupes affectés par des pathologies négligées, confrontés à l'isolement et l'urgence (Callon, \& Rabeharisoa 1998, Epstein 1995, Brown 2007, Akrich 2016). Ils ne réclament pas non plus une «autre » science plus engagée, libérée du carcan de normes jugées trop conservatrices. Au contraire, ils souhaitent 
aider l'IECP à produire des données "incontestables", conformes aux critères académiques en vigueur dont ils espèrent tirer des arguments supplémentaires pour interpeller les autorités à propos des pollutions trop grandes et trop nombreuses qu'ils subissent. Cependant, on peut dire que leur implication, via des expériences de biosurveillance participative, a aussi contribué à modifier les savoirs sur la pollution. Nous y reviendrons après avoir évoqué le rôle qu'ont tenu les lichens dans cette transformation.

\section{Les lichens comme « hygiomètres » de la qualité de l'air}

Différentes espèces végétales et animales sont mobilisées depuis longtemps pour détecter les signes avant-coureurs des dangers, notamment depuis que la révolution industrielle a multiplié les situations à risque. Par exemple, on sait qu'à partir de la fin $\mathrm{du}$ XIX $\mathrm{X}^{\mathrm{e}}$ siècle, des canaris étaient descendus dans les puits de mine pour prévenir les accidents. Cette pratique semble s'être généralisée suite aux travaux du physiologiste britannique John Scott Haldane sur les effets des gaz toxiques sur le corps humain (Goodman 2008). Haldane a contribué à la conception de divers équipements de sécurité pour les mineurs et les équipes de sauvetage, telles des lampes à flamme bleue qui permettaient de repérer la présence de monoxyde de carbone. Toutefois, pendant près d'un siècle, les canaris ont été très largement privilégiés pour leur grande sensibilité au $\mathrm{CO}$ et leur capacité à donner l'alerte en cas d'émanations chimiques et/ou explosives. Les faibles coûts associés à leur élevage et leur fiabilité expliquent leur succès.

Les animaux et les plantes, du fait de leur métabolisme, peuvent en effet être de bons informateurs sur l'état de nos environnements et même des sentinelles capables d'annoncer des catastrophes avant qu'elles ne se produisent. Ainsi, à Minamata, c'est chez les chats du port, grands consommateurs de poissons, que les symptômes causés par le méthylmercure se sont d'abord manifestés (permettant ensuite au docteur Hajime Hosokawa d'établir un lien de causalité avec les pathologies des riverains de la baie, bien que ses résultats fussent gardés secrets par l'entreprise qui l'employait, cf. Jobin 2006). Lorsque Rachel Carson publie Printemps silencieux en 1962, souvent présenté comme l'ouvrage fondateur de l'écologie politique aux États-Unis, elle s'est appuyée sur les observations de plusieurs chercheurs, notamment des ornithologues qui, dès les années 1950, avaient constaté une raréfaction des oiseaux communs des villes, des campus et des forêts, et cherché à en connaître la cause. Jusque-là, les recherches avaient surtout porté sur les effets aigus, c'est-à-dire l'impact immédiat des pesticides organochlorés utilisés à haute dose. Carson pointait plutôt du doigt les effets cumulatifs délétères de ces substances à faibles doses ${ }^{9}$.

D'autres expériences ont également été conduites pour tester la capacité des plantes et des animaux à nous guider dans l'évaluation des risques ${ }^{10}$. Les premières concernant l'atmosphère remonteraient au XIX $^{\mathrm{e}}$ siècle ${ }^{11}$. Alors que les effets néfastes de la révolution industrielle sur la qualité de l'air des grandes villes se faisaient sentir, Wilhelm Nylander, un botaniste finlandais travaillant pour le Muséum d'histoire naturelle à Paris a commencé à cartographier la pollution à partir de la distribution des lichens, qu'il qualifiait d'«hygiomètres ${ }^{12}$. Ainsi, lors d'une réunion de la Société botanique de France, il déclarait : 
"Comme les lichens donnent, à leur manière, la mesure de la salubrité de l'air, et constituent (si l'on peut ainsi dire) une sorte d'hygiomètre très sensible, j'ai cru utile de faire un relevé des espèces que j'ai notées dans cette promenade publique [le Jardin du Luxembourg]» (1866:365).

17 Le court débat qui suivit confirme ses observations, un autre botaniste indiquant que la décroissance du nombre de lichens pouvait effectivement être attribuée à la «corruption » de l'air (1866: 371). Les personnes présentes évoquent aussi le noir de fumée des usines qui se dépose sur les arbres du Muséum, mais également un champignon qui pourrait avoir les mêmes effets. La suite de la discussion est remise à plus tard, les membres de la société savante préférant se recentrer sur des questions de taxonomie générale. Nylander, lui-même, n'a pas consacré plus de temps aux qualités « hygiométriques » des lichens. L'essentiel de son travail a consisté à décrire environ deux mille espèces, et à classer des collections pour d'autres, jusqu'à sa mort prématurée, dans la plus grande solitude et les privations qu'il s'imposait (Cartroux 2012). Il faut dire que ce lichénologue obsessionnel s'était fâché à peu près avec tous ses collègues à propos de la nature des lichens. Il arguait qu'ils appartenaient à une catégorie distincte et autonome, contre les idées de symbiose d'algues et champignons, avancées par S. Schwendener, qui allaient s'imposer à l'issue d'une controverse de vingt ans. Son caractère réservé et obstiné, son opposition à ce qu'il nommait le «schendenerisme » reposant selon lui sur une " opinion » et non sur «l'observation » (Honegger 2000), expliquent sans doute qu'il n'ait pas fait « école ». Seul un de ses amis allemands, Ferdinand Arnold, a tenté de transplanter des lichens à Munich pour étudier les effets des pollutions sur leur développement, dans les années 1890, tandis qu'un botaniste suédois, Rutger Sernander, s'attelait à inventorier les espèces présentes dans Stockholm pour en produire une cartographie (Nimis, Scheidegger \& Wolseley 2000, Wilkomirski 2013). Il a donc fallu attendre les années 1960 pour que les lichens et d'autres « organismes bio-indicateurs » soient enfin reconnus comme des objets de recherche légitimes pour la surveillance environnementale (Gilbert 1968, Hawksworth \& Rose 1970). Il semble qu'entre-temps, ce soient plutôt les chimistes qui se sont imposés comme les seuls spécialistes de la métrologie des pollutions (Massard-Guilbaud 2010), proposant des méthodes et des techniques qui firent longtemps autorité, tels Auguste Girardin récompensé par l'Académie des sciences en 1895 pour son «test papier » des fumées (Lestel 2013).

18 Aujourd'hui, les lichens sont considérés comme de bonnes sentinelles de la qualité de l'air (Agnan 2013). Leurs qualités sont attestées par une littérature scientifique abondante. Comme ils n'ont pas de racine, ces symbioses d'algues et de champignons dépendent entièrement des apports de l'atmosphère dont ils tirent tous leurs moyens d'existence. Ils peuvent dès lors nous fournir des informations exclusives sur les caractéristiques de cette dernière (alors que des organismes plus complexes comme les arbres sont soumis à influences, celles des sols ou de l'eau qu'il est compliqué de discriminer). Malgré cette relative simplicité, leur petite taille, leur capacité à épouser les formes des supports qu'ils colonisent et habillent jusqu'à les camoufler et se confondre avec eux tout à la fois ${ }^{13}$, les lichens fascinent. Des recherches récentes montrent qu'ils pourraient être immortels, si les conditions ne leur sont pas défavorables (Pringle 2017). On trouve des dizaines de milliers d'espèces différentes. Ils croissent dans la plupart des régions du monde (chaudes, froides, arides ou humides), sur des substrats très hétérogènes et excessivement pauvres. Les lichens ont toutefois 
des préférences. Certains sont plus polluotolérants (ou polluosensibles) que d'autres. Leur présence (ou absence), la composition des populations expriment à elles seules des indices utiles pour qui sait les repérer et les interpréter ${ }^{14}$. Ils permettent d'évaluer rapidement la qualité écologique d'un site. Ces capacités «bioindicatrices» sont d'ailleurs officiellement reconnues, au-delà des seules arènes scientifiques. Elles ont donné lieu, en France, à la définition de méthodes normalisées pour l'observation et l'interprétation de la diversité lichénique (AFNOR 2014 - NF 16413), mais aussi de méthodes d'échantillonnage adaptées pour la biosurveillance de la qualité de l'air (AFNOR 2013 - NF X43-904) dans les années 2010. La législation requiert d'ailleurs des industriels qu'ils complètent les mesures de leurs rejets dans l'environnement par des dispositifs mobilisant des espèces sentinelles comme les lichens, notamment pour les substances comme les dioxines pour lesquelles il n'existe pas de techniques automatisées en continu. À Fos, des essais ont été réalisés avec l'incinérateur de déchets industriels Solamat-Merex, y compris pour les métaux, mais avec des résultats mitigés. Les premières analyses de 2013 ont conclu qu'en raison de la pluralité des sources de pollution, il était difficile de se prononcer sur les responsabilités spécifiques de l'entreprise commanditaire ${ }^{15}$.

\section{Des agencements multispécifiques pour connaître les effets des pollutions}

Les scientifiques de l'IECP, au contraire, s'intéressent aux lichens dans le but de produire des données globales, pertinentes pour le territoire, sur les effets cumulés des pollutions. Ils souhaitent améliorer et pousser plus loin encore la surveillance réglementaire, soit les prélèvements en routine des stations d'AIRPACA et les mesures en autocontrôle des industriels, simultanément très localisés et éparpillés. Il s'agit aussi pour eux de pallier les ignorances en menant de véritables recherches. Les lichens présentent l'avantage d'être présents naturellement à peu près partout, offrant ainsi la possibilité de réaliser un suivi étendu mais aussi homogénéisé, puisqu'une seule catégorie de "sentinelle" est mobilisée pour documenter la situation environnementale de toute la ZIP de Fos et ses environs. Des comparaisons dans l'espace et le temps deviennent alors possibles. D'abord aidé par un bureau d'études (Aair lichens), le personnel scientifique de l'IECP a donc fait des repérages. Il a fallu définir des « placettes » d'observation. Une annonce visant à recruter des volontaires a été publiée. Des riverains ont été recrutés puis formés, le temps d'un week-end, à l'occasion d'un cours théorique puis d'une sortie sur le terrain. Le protocole de bioindication a été modifié et adapté pour les volontaires, qui ne sont pas équipés de loupes binoculaires ou de microscopes, par un jeune chercheur spécialiste des lichens, recruté pour l'occasion. Un dossier photographique ainsi qu'une clé de détermination simplifiée (créée à dessein pour faciliter l'implication d'acteurs non-professionnels) leur ont été remis. Il a ensuite été demandé aux VOCE d'effectuer des relevés annuels sur les quatre faces de cinq arbres à l'aide d'une grille d'observation composée de cinq mailles de $10 \times 10 \mathrm{~cm}$ superposées verticalement, à au moins un mètre de hauteur (Photos 2, 3). Chaque opération a été prévue pour se dérouler en 2 temps:

- identification des morphologies lichéniques (Crustacés, Foliacés et Fruticuleux) ;

- reconnaissance de 4 espèces de lichen sélectionnées pour leur présence et polluosensibilité. 
19 Les volontaires relèvent la présence ou l'absence des lichens préalablement ciblés, puis estiment leur abondance sur une échelle de valeur de 0 à 5 . Cette évaluation est reportée dans un tableau (Photo 4).

\section{Démonstration lors de la formation des VOCE à l'observation lichénique}

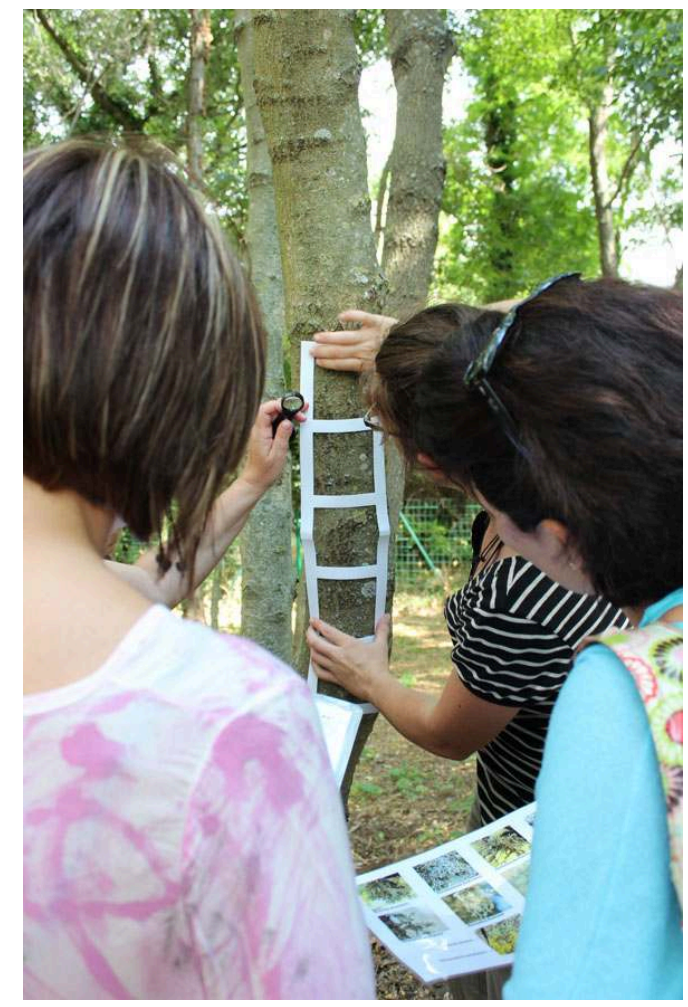

IECP / C. Gramaglia 
3. Démonstration lors de la formation des VOCE à l'observation lichénique

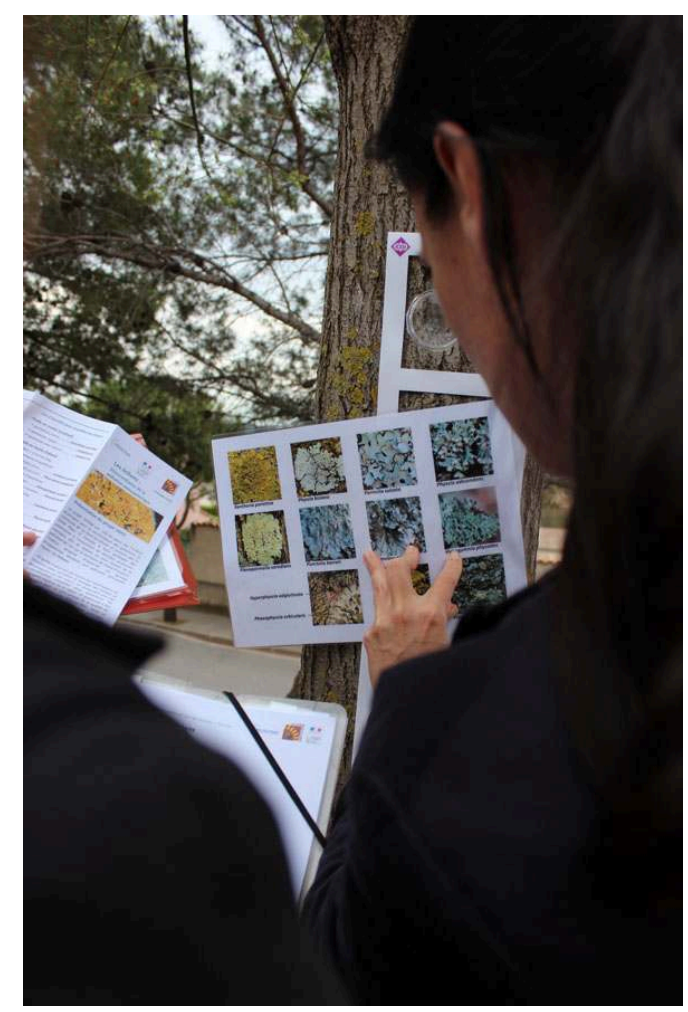

IECP / C. Gramaglia

4. Un volontaire désigne l'échelle de valeur

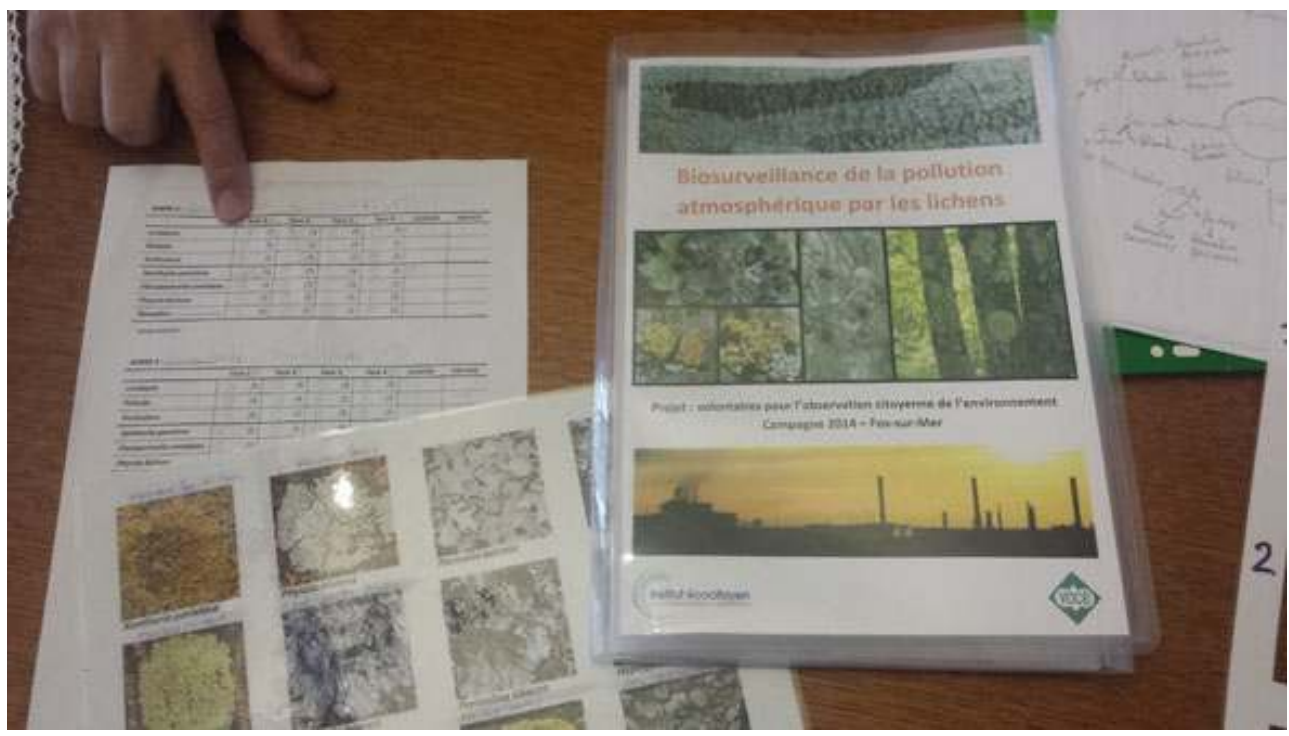

C. Gramaglia

Les données recueillies, géoréférencées, sont transmises au personnel scientifique de l'IECP, lequel procède à leur traitement. Les relevés des VOCE permettent d'avoir une première vue d'ensemble sur la diversité lichénique et donc la qualité de l'air. Si des modifications importantes apparaissent d'une année à l'autre, l'IECP peut intervenir rapidement et lancer des investigations plus poussées en matière de bioindication et de 
bioaccumulation, seules capables de quantifier une augmentation de la pollution. Pour ce faire, divers instruments mathématiques validés par la littérature sont mobilisés, soit l'indice de diversité Shannon, soit l'indice de pureté atmosphérique, deux équations qui corrèlent, pour l'une, la richesse spécifique à leur répartition, et pour l'autre, le nombre d'espèces observées à leur classification écologique, celle-ci rapportée à un coefficient de recouvrement révélateur de l'abondance et de la densité. Il importe alors d'inventorier toutes les espèces avec précision, ce qui nécessite d'avoir une connaissance affinée des taxons et du matériel de visualisation supplémentaire (microscope, loupe mais aussi produits ou réactifs chimiques tels que ceux que W. Nylander testait déjà pour discriminer les espèces, cf. Vitikainen 2001). Seul le personnel scientifique de l'IECP est en capacité de le faire, soit à l'occasion de campagnes spécifiques prévues d'une année sur l'autre, soit ponctuellement, après un signalement des volontaires.

21 Les résultats des toutes premières études menées par l'IECP avec les VOCE montrent que les lichens les plus fréquents dans la ZIP de Fos sont des Xanthoria parietina (photo 5), connus pour leur tolérance aux pollutions, avec une dizaine d'autres espèces aux caractéristiques similaires (Dron et al. 2016).

\section{Une espèce résistante à la pollution (Fos) : Xanthoria parietina}

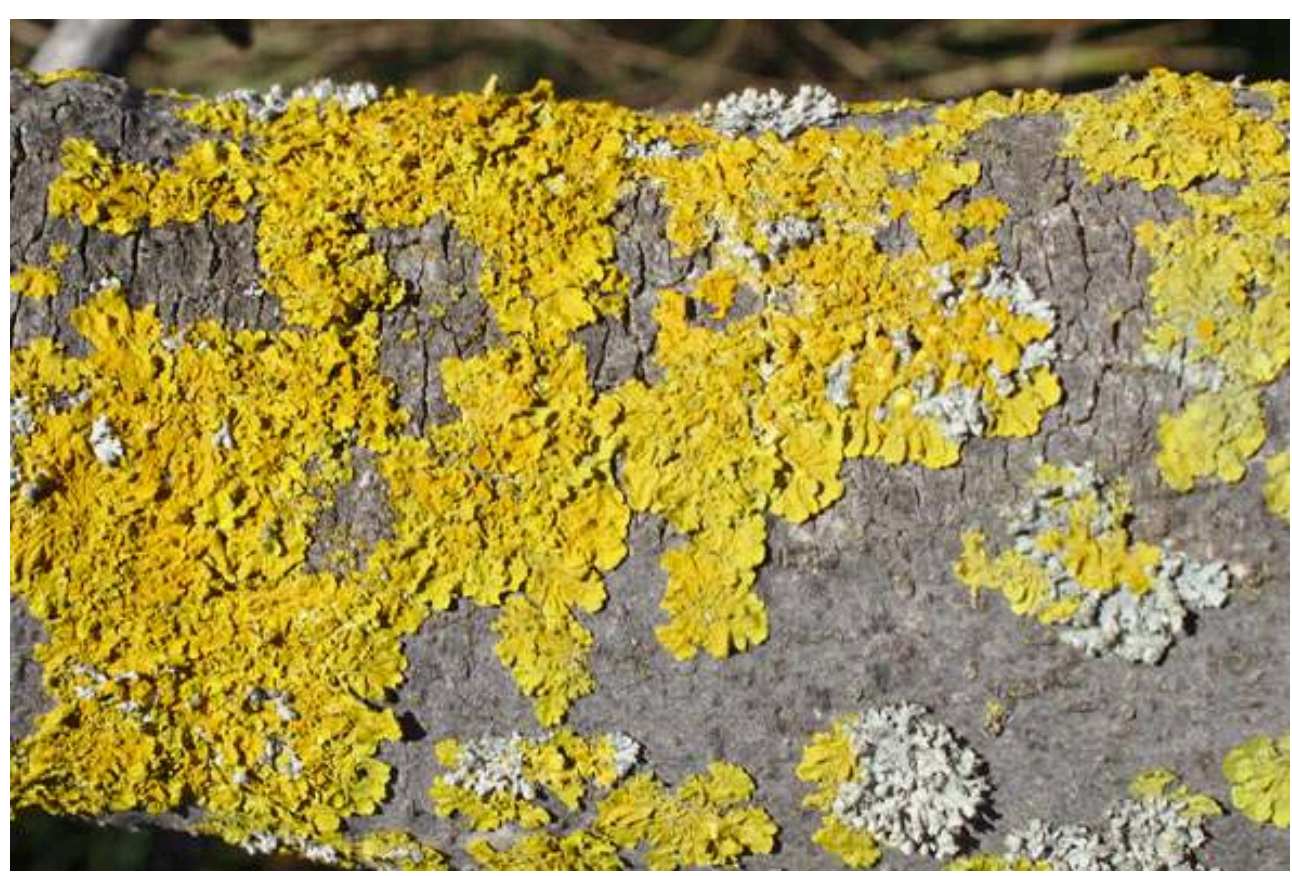

IECP

22 Par contre, dès qu'on s'éloigne des installations industrielles, la diversité lichénique augmente pour atteindre une vingtaine d'espèces dans une « placette » témoin à Grans. Cette dernière comprend d'ailleurs des espèces particulièrement sensibles à la pollution atmosphérique et donc plus rares comme Ramalina farinacea (photo 6) à la physionomie beaucoup plus échevelée et aérienne que la robuste Xanthoria parietina, tapie comme un crustacé sur l'écorce des arbres. 


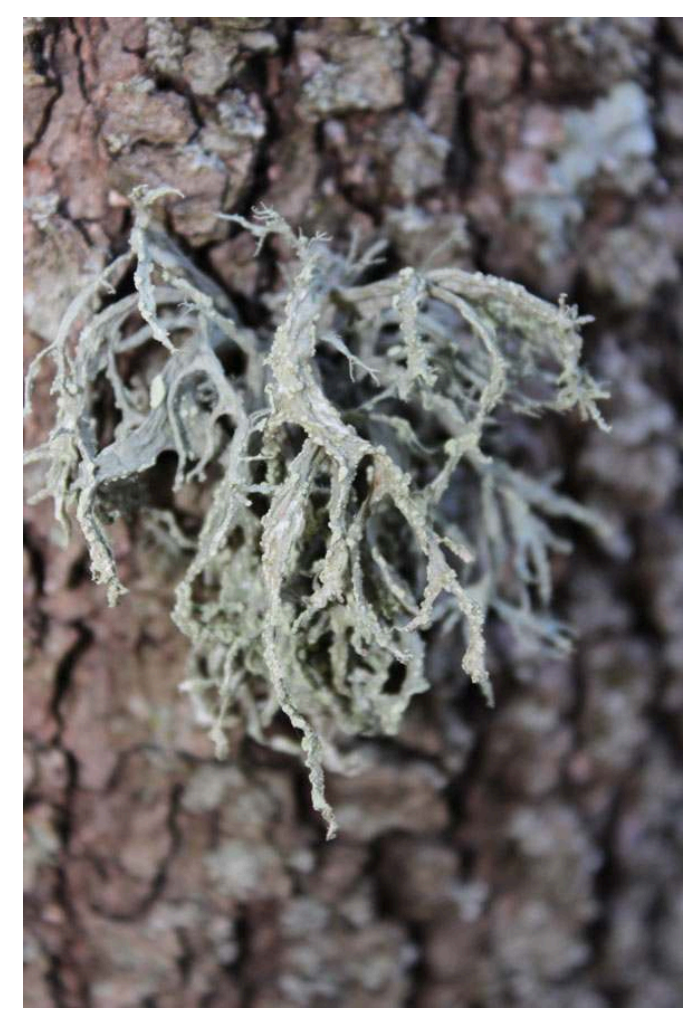

IECP

23 Ces observations rendent compte d'un gradient de pollution manifeste. La diversité lichénique décroît en effet de façon régulière quand on se rapproche de la ZIP de Fos ou $\mathrm{du}$ complexe pétrochimique de Lavéra voisin. Les formes de lichens y sont moins exubérantes, comme si le développement des espèces foliacées ou fruticuleuses y avait été empêché. De même, les tests auxquels le personnel scientifique de l'IECP soumet ses échantillons donnent une idée précise de leurs niveaux de contamination par exemple, aux métaux, hydrocarbures aromatiques polycycliques, polychlorobiphényles ou dioxines. Les spécimens prélevés sur les arbres sont séchés et broyés, puis envoyés pour analyse dans un laboratoire disposant d'un chromatographe en phase gazeuse couplée à un spectromètre de masse, soit le Laboratoire de la faculté de pharmacie de Marseille, soit un laboratoire agréé.

Les résultats des études menées entre 2011 et 2015 pointent ainsi des teneurs de 10 à 100 fois supérieures pour certains métaux et des hydrocarbures à proximité des installations industrielles, dans des zones proches des habitations. Les résultats sont cependant moindres voire peu significatifs pour les dioxines. L'ensemble de ces données à la fois scientifiques et citoyennes, parce que produites de manière participative, permet de "tenir un registre des pollutions » dans le temps et l'espace, selon les propos du directeur de l'IECP livrés en entretien. Le rôle qu'y tiennent les lichens n'est pas secondaire: ils conditionnent la participation. Il ne s'agit pas d'« intermédiaires ", mais plutôt de "médiateurs » de l'action (Latour 2006), c'est-àdire qu'ils produisent une transformation ou une information nouvelle. Ici, la présence ou l'absence de certains lichens, la composition des colonies, les préférences ainsi révélées, nous renseignent efficacement sur les qualités de leur environnement, ou plutôt sur ce qui compte le plus pour eux, autrement dit, les qualités de l'air dont ils 
dépendent entièrement (et avec qui ils entretiennent une relation exclusive). Leur indifférence à d'autres éléments renforce leur acuité à propos de l'atmosphère. Il suffit de connaitre leurs exigences et d'y prêter attention. Un inventaire rapide permet alors d'obtenir, à lui seul, une première estimation, de manière relativement simple et peu coûteuse. À titre de comparaison, il faudrait disposer de plusieurs préleveurs très onéreux pour chaque substance toxique, disposés en plusieurs points du territoire pour assurer un suivi de même ampleur.

Pour l'IECP, il était donc pertinent et utile de tisser des liens entre ses scientifiques, son réseau de volontaires et ces organismes particuliers, parce qu'ils avaient un intérêt commun évident pour la qualité de l'air. Grâce à ce que l'on peut qualifier d'agencement multispécifique, pour reprendre le qualificatif de S.E. Kirksey et S. Helreich, pour indiquer que nous n'avons pas affaire à un usage instrumental du vivant, mais bien à une forme distribuée d'action impliquant activement les lichens (dont le développement est façonné par des forces biophysiques tout autant que sociopolitiques et peut avoir, en retour, des effets sur elles), l'association a pu réduire les ignorances en matière de connaissance et surveillance environnementale dans les espaces industrialisés et habités de la ZIP de Fos. Penser avec ces petits êtres lui permet désormais aussi d'accéder aux "mondes infimes » des effets cumulés et synergétiques des pollutions sur les organismes vivants, quels que soient les émissions, les normes et les seuils réglementaires. Elle se sent désormais suffisamment armée pour traduire politiquement ses observations, étayées par des preuves convergentes acquises de différentes manières. Elle peut ainsi se permettre, dans le résumé d'un article scientifique que des membres de son personnel scientifique ont fait paraitre dans une revue à comité de lecture, de pointer : "l'inadéquation de la réglementation appliquée par les industriels... qui se cantonne généralement au voisinage immédiat de leurs installations » et "le besoin d'une surveillance intégrée sur un territoire défini de manière cohérente avec son usage et ses spécificités et incluant les zones d'habitation qui doivent même figurer au centre de la biosurveillance atmosphérique » (Dron et al. 2016 : résumé).

Toutefois, à la transformation du processus de production des savoirs et des connaissances sur la pollution elles-mêmes, s'ajoutent des changements supplémentaires. Penser avec les lichens, semble avoir entraîné les volontaires plus loin encore. Il ne s'agissait pas tant pour eux de participer à un processus d'accumulation de données, que de s'engager dans une activité collective jugée utile, dans le contexte défavorable que nous avons décrit plus haut. L'identification des sources de pollution et des points chauds de la contamination a satisfait la plupart. Beaucoup font d'ailleurs état d'un intérêt pour les résultats détaillés plus faible que ce que nous avions imaginé. Ce qui est surprenant aussi, c'est de constater que les lichens, ces très petits organismes qui ne portent ni fleur ni fruit, parfois qualifiés d'« inférieurs » malgré la complexité des dynamiques symbiotiques qui les constituent, ont pu modifier l'expérience des VOCE. Ils ne faisaient l'objet d'aucun usage commun dans le territoire de Fos-sur-Mer avant les recherches de l'IECP, bien qu'y étant répandus (et même physiquement attachés, comme les riverains de la ZIP de Fos peuvent l'être affectivement). Ils n'étaient qu'exceptionnellement remarqués par les habitants de la ZIP de Fos et ses environs. Après la formation des volontaires et les premières campagnes d'observation participatives, les choses ont changé. Plusieurs de ceux que nous avons rencontrés nous ont expliqué qu'ils les "voyaient » enfin, mais surtout que le regard qu'ils portaient sur leur territoire s'était modifié grâce à eux. David participant engagé et appliqué, nous a fait remarquer qu'il appréciait désormais 
les «jolies couleurs » des lichens sur lesquelles il s'arrêtait occasionnellement quand il montait sur son toit ou se baladait en forêt. L'expérience d'Olivier nous ouvre quant à elle d'autres pistes de réflexion concernant la puissance desagencements multispécifiques. C'est un volontaire qui a suivi la formation à la biosurveillance lichénique, mais n'a jamais voulu y participer. Il a préféré contribuer à des suivis du milieu marin, du fait de sa passion trop forte pour la plaisance. Par contre, il nous explique comment l'attention nouvelle qu'il porte aux lichens, presque malgré lui, influence sa façon d'estimer les qualités d'un espace, en particulier son environnement. Son expérience en est bouleversée. Il ne semble plus capable de faire comme avant, c'est-à-dire sans leur compagnonnage, pour apprécier d'autres espaces et donner sens aux perceptions qu'il peut en avoir.

«J'ai bien pris note que l'on voyait les pollutions sur les lichens, j'ai été étonné d'ailleurs de voir ça. On a visité tous les arbres, c'était très bien fait. Mais je ne voyais pas personnellement mon intérêt d'aller dedans, je préférais plutôt m'orienter sur ce que j'aime le plus, c'est-à-dire la mer, j'étais plutôt branché mer... Mais, c'est vrai que quand je vois un arbre maintenant, je regarde un petit peu. On est allé à Cuba il y a pas longtemps, et c'est vrai que j'ai vu des arbres qui étaient couverts de lichens. Alors qu'ici il y en a très peu... Bon, il y a l'ambiance, c'est tout à fait différent. C'est une île et tout. Mais, ça m'a étonné de voir des arbres tout verts avec de beaux lichens en relief et tout, alors qu'ici il y en a très peu. Je sais que quand il y a pas de lichen, c'est grave [rire]... Ça m'a permis, voyez... J'ai vu que là-bas, il y avait beaucoup moins de pollution. C'est tout. Autrement, comme je vous l'ai dit, vu que les arbres ici à part ceux qu'on a plantés, il n'y en a pas beaucoup, et que sur ceux qu'on a plantés, il n'y a pas de lichen... ».

Le terme de compagnonnage (Haraway 2008) nous semble particulièrement pertinent ici parce qu'il permet de distinguer le mode relationnel qui unit scientifiques, volontaires et lichens, mieux que celui de «travail » mobilisé à propos d'autres espèces non-humaines, par exemple les chiens guides d'aveugles ou de compagnie, ou encore les abeilles entrainées à repérer des explosifs et armes chimiques à Fort Alamos, aux USA (Kosek 2010). Ceux-ci n'exercent pas d'activité à proprement parler pour les VOCE ou les chercheurs de l'IECP. Cependant, leur présence (ou absence) correctement appréciée devient une source d'information précieuse. L'expérience est gratifiante pour les volontaires qui ont l'impression de contribuer à l'effort de recherche collectif en faveur du territoire (et utile aux scientifiques qui n'ont pas la possibilité de le parcourir régulièrement). Elle fournit bien évidemment des données qui pourront étayer des revendications collectives. Elle permet également de lutter contre les effets corrosifs des pollutions sur le tissu social (Freudenburg 1997) qui tendraient à éloigner les populations riveraines des usines, de leurs semblables, mais aussi de leur environnement jugé dégradé, parfois jusqu'à l'anomie. L'observation qui nécessite une forme d'attention particulière, signe aussi l'émergence d'une communauté de destin entre humains et non-humains (Descola 2005) confrontés de concert aux pollutions. Le fait qu'elle soit initiée à partir d'un petit être vivant avec lequel nous n'avons a priori rien à voir et pour lequel aucune sympathie préalable ne s'était exprimée est à même de bousculer nos façons de penser. Il ne s'agit pas de sauver une espèce emblématique en danger, mais d'apprendre d'elle et surtout de penser « avec » elle les relations qui constituent les écosystèmes dans lesquels nous sommes communément plongés. On n'est pas dans un rapport instrumental, comme avec un "sceau ", si on se réfère aux mobilisations nord-américaines qui relèvent aussi de la contre-surveillance 
environnementale. Les VOCE, une fois formés, développent une capacité d'attention accrue qui les sensibilise tout autant qu'elle les rend aptes à identifier, seuls si besoin, où ils se trouvent et sans équipement spécifique s'ils n'en ont pas sous la main, des signes de bonne santé ou de perturbation environnementale. Les lichens deviennent leur compagnon interprète en la matière. Cette vigilance distribuée nouvelle, in situ, est aussi une expérience qui désanthropocentrise pour mieux politiser les phénomènes infimes qui peuvent affecter le monde commun et qui sont trop souvent réduits à des questions techniques. Des attachements riches de sens et porteurs de transformations sont alors possibles qui ouvrent la voie à des préoccupations et des formes de sollicitude multispécifiques (de la Bellacasa 2012), lesquelles, nous en faisons l'hypothèse, sont indispensables à ceux qui posent un regard critique sur l'habitabilité du golfe de Fos et réfléchissent aux façons de la restaurer, imaginant déjà des « arts de vivre » dans les ruines du capitalisme industriel (Tsing et al. 2017).

La pollution peut être considérée comme un «hyperobjet » au sens de Timothy Morton (2013), en cela qu'elle a la capacité de contaminer les matérialités et les organismes vivants avec lesquels elle entre en contact, mais qu'elle échappe aussi à la plupart des efforts déployés pour l'appréhender, la décrire et la contrôler. Majoritairement invisible, mouvante, et inégalement distribuée dans l'espace, elle est aussi plurielle car résultant de l'interaction de plusieurs substances et phénomènes. Elle relève en grande partie des «mondes infimes » (celui des substances chimiques, leurs effets cumulés et synergétiques), malgré son volume. L'impossibilité de la saisir immédiatement en totalité, nous oblige donc à croiser des méthodes diverses et complémentaires pour en identifier des traces, l'évaluer et la surveiller, à partir de perspectives humaines et nonhumaines différentes mais complémentaires. Il ne s'agit pas d'un problème d'échelle (Latour 2014, Houdart \& Manceron 2015). Le point de vue des lichens n'est pas celui des riverains de la ZIP, en plus petit. Il est autre, néanmoins significatif.

Les recherches scientifiques et citoyennes lancées par l'IECP sont autant de réponses stratégiques à ces difficultés, particulièrement pour ce qui concerne la biosurveillance scientifique et citoyenne dont l'objectif est de produire des connaissances pour, in fine, changer la surveillance réglementaire. En complément d'instruments de laboratoire usuels, performants mais très coûteux, l'association a recours à plusieurs organismes vivants comme les lichens pour obtenir des informations sur l'état général de l'environnement à Fos-sur-Mer et Port-Saint-Louis du Rhône. Celles-ci, une fois agrégées, la renseignent utilement sur la qualité de l'air et lui permettent d'estimer, assez rapidement, les conséquences de la pollution atmosphérique (approchée justement en croisant le point de vue de plusieurs espèces "sentinelles»). Outre les lichens, l'IECP mène des expériences sur les pétunias et les salades avec son réseau de volontaires. D'autres organismes pourraient être mobilisés dans le futur, selon les besoins (mais aussi les intérêts des VOCE dont on a vu qu'ils importaient grandement, ceux-ci ayant du mal à s'engager durablement dans des opérations qui ne coïncident pas avec leurs passions). Le personnel scientifique de l'IECP est dans la quête continue de nouveaux médiateurs susceptibles de lui donner accès à des données inédites qui «parlent du territoire » en même temps que de la pollution, l'expression leur revient, et l'autorisent à penser qu'il sera possible de faire évoluer la réglementation en apportant les preuves incontestables de ses limites (notamment l'inadaptation des normes et des seuils standards dans un territoire aussi particulier qu'une ZIP). 

scientifique et social revendiqué par l'IECP. La littérature qui porte sur les sciences citoyennes se concentre plutôt sur des suivis participatifs de la biodiversité à des fins de connaissance des milieux et espèces, tandis que celle qui traite de la surveillance ou plutôt contre-surveillance des pollutions s'appuie majoritairement sur des cas où des instruments de mesure bricolés, des appareils automatiques ou de nouvelles technologies sont mobilisés dans un but protestataire. À notre connaissance, le rôle des non-humains y est passé sous silence. Dans ce contexte, les recherches menées à Fossur-Mer apparaissent comme une expérience de pensée inédite « avec » des organismes vivants, dans le cadre d'agencements multispécifiques riches de perspectives à la fois cognitives, sociopolitiques et affectives (Haraway 2015). La production de connaissances nouvelles, solides et localement pertinentes est revendiquée, comme une véritable science "par le bas", c'est-à-dire plus juste parce que tenant compte des besoins des populations concernées (Harding 2008). Ce qualificatif pourrait également signifier qu'elle se fait à partir de la perspective d'organismes de petite taille. Dès que la connexion est établie entre les lichens et les volontaires, nous l'avons montré, elle devient opérante. Le doute s'immisce: l'initiative en revient-elle aux humains qui portent leur regard sur les lichens ou les lichens obligent-ils les humains à revoir leur point de vue ? Cette question, inspirée par les travaux de Michaël Pollan (2001) et de Natasha Myers (2015), renvoie à notre propre aptitude à nous laisser interpeller par eux. Les pratiques et les perceptions des VOCE rencontrés ont bien été modifiées. Les lichens sont devenus pour eux comme des compagnons disséminés dans l'espace (à la différence peut-être d'autres organismes vivants cultivés ou moins généreusement répartis à l'origine), capables de faire "toucher du doigt la pollution", et même d'indiquer où et comment habiter un environnement altéré par les excès du capitalisme (Tsing 2015, Murphy 2016), éventuellement comment restaurer un peu de son habitabilité perdue. À condition de porter attention à ce qui compte pour eux, de regarder notre monde commun à partir de leur perspective, aussi petite nous semble-telle.

\section{RÉFÉRENCES}

\section{BIBLIOGRAPHIE}

Abbé Hue, M. L. 1899 William Nylander. Bulletin de la Société Botanique de France 46 (3) : 153-165.

Abram, D. 2013 Comment la terre s'est tue : pour une écologie des sens. Paris : La Découverte.

Agnan, Y. 2013 Bioaccumulation et bioindication par les lichens de la pollution atmosphérique actuelle et passée en métaux et en azote en France : sources, mécanismes et facteurs d'influence. Thèse en sciences de l'univers, de l'environnement et de l'espace. Université Toulouse.

Akrich M. 2016 « Inquiries into experience and the multiple politics of knowledge. Keynote Plenary 1 : To what extent is embodied knowledge a form of science and technology by other means? » 4S-EASST Meeting. Barcelona : CSI Working paper. 
Akrich, M., Rémy, C. \& Y. Barthe 2013 Sur la piste environnementale: Menaces sanitaires et mobilisations profanes. Paris : Presses des Mines.

Alphandéry, P. \& A. Fortier 2013 « Les données sur la nature entre rationalisation et passion », Revue du MAUSS 2 : 202-220.

Batty, L. C., \& K. B. Hallberg (dir.) 2010 Ecology of industrial pollution. Cambridge University Press. Blanc, N. 2010 « De l'habitabilité urbaine » in O. Coutard, J.-P. Levy (dir.) Écologies urbaines. Paris : Économica.

Blanchard, F., Hube, D., Salquebre, D., Allier, D., Saffre, A.-S. et al 2009 Aigrette. Synthèse des résultats et plan d'action à l'échelle du territoire du San Ouest Provence. Rapport final, BRGM.

Blondiaux, L. \& J.-M. Fourniau 2011 « Un bilan des recherches sur la participation du public en démocratie : beaucoup de bruit pour rien ?", Participations $1:$ 8-35.

Brown, P. 1997 « Popular epidemiology revisited », Current Sociology 45 (3) : 137-156.

Callon, M. 1986 « Éléments pour une sociologie de la traduction : la domestication des coquilles Saint-Jacques et des marins-pêcheurs dans la baie de Saint-Brieuc ", L'Année sociologique (1940/1948) $36: 169-208$.

Callon, M., Lascoumes, P. \& Y. Barthe 2001 Agir dans un monde incertain. Essai sur la démocratie technique. Paris : La Découverte.

Cartoux, E. 2012 Les lichens de William Nylander. Muséum d'histoire naturelle Henri-Lecoq : Clermont-Ferrand.

Charvolin, F., Micoud, A., \& L. K. Nyhart (dir.) 2007 Des sciences citoyennes ? La question de l'amateur dans les sciences naturalistes. Paris : Éditions de l'Aube.

Chaudhary, R. P., Werth, S. \& C. Scheidegger 2017 «Indigenous knowledge and use of lichens by the lichenophilic communities of the Nepal Himalaya ", Journal of Ethnobiology and Ethnomedicine 13 : 15. doi: 10.1186/s13002-017-0142-2.

Chateauraynaud, F. \& D. Torny 2005 « Mobiliser autour d'un risque. Des lanceurs aux porteurs d'alerte ». [En ligne] : halshs.archives-ouvertes.fr/halshs-00411847/document.

Clarke, A. \& J. Fujimura 1996 La Matérialité des Sciences Savoir-Faire et instruments dans les Sciences de la Vie. Paris : La Découverte.

Daumalin, X. \& C. Gramaglia [à paraître] « "Ni partir, ni mourir, mais vivre ici”. Jalons pour une sociohistoire des mobilisations contre les pollutions dans la zone industrialo-portuaire de Berre/ Fos-sur-mer ", Actes du colloque Santé et environnement. Parcours et constructions historiques. Éditions des archives nationales de Pierrefitte.

Bellacasa, M. P. de la 2012 " "Nothing comes without its world": thinking with care ", The Sociological Review 60 (2) : 197-216.

Descola, P. 2005 Par-delà nature et culture. Paris : Gallimard.

Dron, J., Austruy, A., Agnan, Y., Ratier, A. \& P. Chamaret 2016 « Utilisation de la biosurveillance lichénique sur la zone industrialo-portuaire de Fos-sur-Mer : retour sur trois ans de suivi à l'échelle d'un territoire intercommunal », Pollution atmosphérique : 2268-3798.

Ellis, R. \& C. Waterton 2005 « Caught between the cartographic and the ethnographic imagination : the whereabouts of amateurs, professionals, and nature in knowing biodiversity ", Environment and Planning D : Society and Space 23 (5) : 673-693. 
Epstein, S. 1995 « The construction of lay expertise : AIDS activism and the forging of credibility in the reform of clinical trials ", Science, Technology \& Human Values 20 (4) : 408-437.

Freudenburg, W. R. 1997 « Contamination, corrosion and the social order : An overview », Current Sociology 45 (3) : 19-39.

Frickel, S. \& M. Edwards 2013 « Untangling Ignorance in Environmental Risk Assessment » in S. Boudia \& N. Jas dir. Toxicants, Health and Regulation since 1945. London : Pickering \& Chatto : 215-233.

Frickel, S., Gibbon, S., Howard, J., Kempner, J. et al. 2010 « Undone Science : Charting Social Movement and Civil Society Challenges to Research Agenda Setting ", Science Technology Human Values 35 : 444-473.

Gilbert, O. L. 1968 « Bryophytes as indicators of air pollution in the Tyne Valley ", New Phytologist 67 (1) : 15-30.

Girard, T. 2012 Les pouvoirs du danger. Zone industrielle de Fos-sur-Mer. Anthropologie politique des risques industriels et du conflit de l'incinérateur. Thèse en anthropologie sociale. Paris : EHESS.

Goodman, M. 2008 Suffer and Survive : Gas Attacks, Miners' Canaries, Spacesuits and the Bends : the Extreme Life of Dr. JS Haldane. Pocket Books.

Gramaglia, C. 2009 « Passions et savoirs contrariés comme préalables à la constitution d'une cause environnementale ", Revue d'anthropologie des connaissances 3 (3) : 406-431.

Gramaglia, C. \& D. Da Silva 2011 « Des mollusques pour « faire parler » les rivières ? » in S. Houdart \& O. Thiéry dir. Humains, non-humains. La Découverte : 221-233.

Haraway, D. 2008 When species meet. University of Minnesota Press.

- 2015 « Anthropocene, capitalocene, plantationocene, chthulucene : Making kin », Environmental Humanities 6 (1) : 159-165.

Harding, S. 2008 Sciences from below : Feminisms, postcolonialities, and modernities. Duke University Press.

Hawksworth, D. L. 1970 « Qualitative scale for estimating sulphur dioxide air pollution in England and Wales using epiphytic lichens », Nature 227 : 145-148.

Honegger, R. 2000 « Simon Schwendener (1829-1919) and the dual hypothesis of lichens ", The Bryologist 103 (2) : 307-313.

Houdart, S., Manceron, V. \& S. Revet 2015 « Connaître et se prémunir. La logique métrique au défi des sciences sociales », Ethnologie française 45 (1) : 11-17.

Irwin, A. 1995 Citizen science : A study of people, expertise and sustainable development. Psychology Press.

Jalbert, K. 2016 « Building Knowledge Infrastructures for Empowerment : A Study of Grassroots Water Monitoring Networks in the Marcellus Shale », Science \& Technology Studies 29 (2) : 26-43.

Jalbert, K. \& A. J. Kinchy 2016 « Sense and influence : environmental monitoring tools and the power of citizen science ", Journal of Environmental Policy \& Planning 18 (3) : 379-397.

Jobin, P. 2006 Maladies industrielles et luttes syndicales au Japon. Paris : Éditions de l'EHESS.

Jouzel, J. N. \& P. Lascoumes 2011 « Le règlement REACH : une politique européenne de l'incertain. Un détour de régulation pour la gestion des risques chimiques », Politique européenne 1 : 185-214. 
Kinchy, A., Parks, S. \& K. Jalbert 2015 « Fractured knowledge : Mapping the gaps in public and private water monitoring efforts in areas affected by shale gas development ", Environment and Planning C : Government and Policy 34 (5): 879-899.

Kirksey, S. \& S. Helmreich 2010 « The emergence of multispecies ethnography », Cultural anthropology 25 (4) : 545-576.

Kosek, J. 2010 « Ecologies of empire : on the new uses of the honeybee », Cultural Anthropology 25 (4) : 650-678.

Lash, S., Szerszynski, B. \& B. Wynne (dir.) 1996 Risk, environment and modernity : towards a new ecology. London : Sage.

Latour, B. 2001 L'espoir de Pandore : pour une version réaliste de l'activité scientifique. Paris : La Découverte.

-2014 « Anti-Zoom » in Olafur Eliasson, Contact. Paris : Flammarion : 121-124.

Lerner, S. 2010 Sacrifice zones : the front lines of toxic chemical exposure in the United States. MIT Press. Lestel, L. 2013 « Pollution atmosphérique en milieu urbain : de sa régulation à sa surveillance », Vertigo-la revue électronique en sciences de l'environnement 15 : https://vertigo.revues.org/12826.

Massard-Guilbaud, G. 2010 Histoire de la pollution industrielle : France, 1789-1914. Paris : Éditions de l'EHESS.

Millot, G., Neubauer, C. \& B. Storup 2013 La recherche participative comme mode de production de savoirs. Un état des lieux des pratiques en France. Paris : Fondations Sciences Citoyennes.

Morton, T. 2013 Hyperobjects : Philosophy and Ecology after the End of the World. University of Minnesota Press.

Murphy, M. 2016 « Expansive Affinities, Anti-affinities, and Industrial Chemical Alterlife, Keynote Plenary 1 : To what extent is embodied knowledge a form of science and technology by other means? »4S-EASST Meeting. Barcelona : CSI Working paper.

Myers, N. 2015 « Conversations on Plant Sensing », Nature Culture $3:$ 35-66.

Nimis, P. L., Scheidegger, C. \& P. A. Wolseley 2002 Monitoring with Lichens-Monitoring Lichens. Springer Netherlands.

Nylander, M. W. 1866 « Les lichens du Jardin du Luxembourg », Bulletin de la Société botanique de France 13 (7) : 364-371.

Osadtchy, C. 2015 Conflits environnementaux en territoire industriel : réappropriation territoriale et émergence d'une justice environnementale : le cas de l'étang de Berre et de Fos-sur-Mer. Thèse de doctorat en géographie. Université du Maine.

- 2016 « Mesurer la pollution : de la prévention des risques environnementaux à la territorialisation par l'action publique environnementale », Terrains \& travaux (1) : 63-83.

Ottinger, G. 2009 « Buckets of resistance : Standards and the effectiveness of citizen science », Science, technology \& human values 35 (2): 244-270.

-2010 « Constructing empowerment through interpretations of environmental surveillance data ", Surveillance \& Society 8 (2): 221-234.

- 2010 « Epistemic fencelines : air monitoring instruments and expert-resident boundaries ", Spontaneous Generations: A Journal for the History and Philosophy of Science 3 (1) : 55-67.

Paillard, B. 1981 La damnation de Fos. Paris : Seuil. 
Pollan, M. 2001 The botany of desire : a plant's-eye view of the world. Random House Trade Paperbacks.

Pringle, A. 2017 « Establishing new worlds : the lichens of Petersham », in Tsing, A., Swanson, H., Gan, E., Busbandt, N. dir. Arts of living on a damaged planet. University of Minnesota Press : 157-169.

Rabeharisoa, V. \& M. Callon 1998 «L'implication des malades dans les activités de recherche soutenues par l'Association française contre les myopathies ", Sciences sociales et santé 16 (3): 41-65.

Roturier, S. \& M. Roué 2009 « Of forest, snow and lichen : Sámi reindeer herders' knowledge of winter pastures in northern Sweden », Forest Ecology and Management, 258 (9) : 1960-1967.

Roux, J., Charvolin, F. \& A. Dumain dir. 2009 Les « passions cognitives » ou la dimension rebelle du connaître en régime de passion, Revue d'anthropologie des connaissances 3 (3) : 369-385.

Tsing, A. 2012 « Unruly Edges : Mushrooms as Companion Species for Donna Haraway ». Environmental Humanities 1 (1) : 141-154.

- 2015 The mushroom at the end of the world: On the possibility of life in capitalist ruins. Princeton University Press.

Vitikainen, 0. 2001 « William Nylander (1822-1899) and lichen chemotaxonomy », The Bryologist $104(2): 263-267$.

\section{NOTES}

1. Le soutien du Labex DRIIHM du CNRS - Investissements d'avenir via l'OHM Littoral (projet Habirisk 2015-2016) a rendu possible l'enquête de terrain à l'origine de cet article.

2. Plusieurs travaux universitaires évoquent les propriétés nutritionnelles, médicinales et tinctoriales des lichens, de même que leur intérêt pour l'agriculture, comme un mémoire de l'Académie de Lyon datant de 1786 ou encore une thèse de médecine de 1853. Leur usage est cependant resté marginal en Europe, contrairement à d'autres régions froides, où la rareté de la végétation en fait un élément central de la pharmacopée (Chaudhary et al. 2017) ou une source de fourrage (Roturier, Roué 2009).

3. Ces données sur la qualité de l'air en zone urbaine et péri-urbaine complètent les suivis réglementaires des émissions atmosphériques de chaque installation industrielles, sous la forme d'auto-contrôles, supervisés par l'Inspection des installations classées.

4. http://www.airpaca.org/monair/commune/13039; http://www.airpaca.org/monair/ commune/ 13078

5. Document de travail sur la mission d'enquête à Fos-sur-Mer du 20 septembre et $1^{\text {er }}$ octobre 2008, Commission des pétitions, Parlement européen.

6. Ottinger indique que les «brigades » ont pu attirer l'attention de l'EPA sur le problème précis des émissions atmosphériques de méthylethylkétone à Norco (Louisiane), en 1998. Néanmoins, l'auteur insiste pour dire que les processus de décision en matière de régulation environnementale n'ont pas changé.

7. Les expériences de sciences citoyennes européennes sont plus nombreuses dans le domaine de suivi de la biodiversité, mais on y retrouve les mêmes problèmes (Ellis \& Waterton 2005, Alphandéry \& Fortier 2013, Millot et al. 2013).

8. Notre recherche s'est déroulée de 2015 à 2016. Nous avons recueilli plus de cinquante entretiens qualitatifs dont 14 visaient spécifiquement des acteurs locaux concernés par la bioindication, scientifiques proches de l'IECP et volontaires principalement. Notre travail a également consisté à assister aux réunions organisées par cette association, soit avec son Conseil 
scientifique, soit avec les riverains de la ZIP de Fos (membres de VOCE ou public plus large). Des échanges plus informels, mais réguliers, avec les scientifiques de l'IECP nourrissent aussi les réflexions présentées dans cet article, d'ailleurs co-écrit par l'un d'entre eux et une sociologue.

9. Aujourd'hui, les accusations portées à l'encontre d'autres pesticides, suspectés de causer la mort des abeilles, emprunte à des registres similaires qui font le lien avec d'autres périls pouvant également affecter les humains.

10. Nous nous concentrerons ici sur des expériences relatives à la qualité de l'air. Nous ne pouvons pas nous étendre sur d'autres observations qui concernent plutôt l'eau, comme celles du chimiste A. Girardin qui avait aussi élaboré une méthode d'évaluation de la qualité de l'eau basée sur l'observation des mollusques, ou les travaux des botanistes R. Kolkwitz et K.M. Marsson qui se sont intéressés aux plantes et invertébrés aquatiques pour étudier les pollutions de rivières, dès 1908.

11. Même si des auteurs ont indiqué que le lien entre pollution et disparition des lichens avait été fait par Arthur Aikin dès le XviII ${ }^{\mathrm{e}}$ siècle, à l'occasion d'un voyage dans le Nord du Pays de Galles où s'implantaient des mines de cuivre. Cette mention ferait d'ailleurs référence à un poème d'Erasmus Darwin, le grand-père de Charles, médecin, botaniste et poète qui regrettait leur raréfaction (Batty \& Hallberg 2010).

12. Dans l'éloge funèbre qu'il lui a consacré, son élève et un temps ami, l'abbé Hué, note qu'il s'agit d'une « très curieuse étude » $(1899: 158)$.

13. D. Abram, dans son essai sur l'écologie des sens, évoque plusieurs fois les lichens et dit qu'ils « donnent vie aux rochers » (2013).

14. Les pollutions altèrent la photosynthèse et peuvent entraîner la mort des lichens.

15. Cf. le compte-rendu du Comité de suivi de site du 4 juillet 2013.

\section{RÉSUMÉS}

La zone industrialo-portuaire de Fos (ZIP) est l'une des plus grandes d'Europe. Elle a été construite à la fin des années 1960 sur le littoral provençal, en lieu et place de la steppe de la Crau, des prairies, des marais et des étangs cernant les villes de Fos-sur-Mer et Port-Saint-Louis du Rhône. Dès le début, les connaissances sur les impacts cumulés des pollutions y ont fait défaut. C'est sous la pression que les élus locaux ont récemment consenti à soutenir la création d'un organisme de recherche indépendant, l'Institut écocitoyen pour la connaissance des pollutions (IECP). Celui-ci, s'est rapidement lancé dans des expériences de biosurveillance scientifique et citoyenne, notamment via l'observation des lichens dans le territoire de la ZIP et une zone témoin. Les premiers résultats tendent à confirmer l'impact marqué des pollutions. Ils conduisent à aussi réclamer une surveillance renforcée. Outre les bénéfices en termes de connaissances, cet article montre que «penser» les pollutions avec les lichens, petits êtres vivants symbiotiques de champignons et algues physiquement attachés à leur environnement dans le cadre d'agencements multispécifiques, change le regard porté par les citoyens volontaires sur l'habitabilité du monde commun et les façons de remédier à sa dégradation.

The industrial-port area of Fos (ZIP) is one of the largest in Europe. It was built at the end of the 1960s on the Provençal coast nearby the towns of Fos-sur-Mer and Port-Saint-Louis du Rhone. From the beginning, knowledge about the cumulative impacts of pollution has been lacking. It is only under pressure that local elected officials have recently agreed to support the creation of an 
independent research organization, the Ecocitizen Institute for Knowing Pollution (IECP). This organization quickly set up scientific citizen biomonitoring experiments, for instance, through the observation of lichens in the territory of the ZIP and a control area. The first results tend to confirm the strong impact of pollution. They support the claim for a reinforced surveillance. In addition to the benefits in terms of knowledge, this article shows that "thinking" about pollution with lichens, small symbiotic living organisms made of mushrooms and algae physically attached to their environment, in the context of multispecific arrangements, changes the of the volunteer citizens on the habitability of their world and the ways to remedy its degradation.

\section{INDEX}

Mots-clés : pollution, surveillance et contre-surveillance, sciences participatives, bioindication, agencements multispécifiques, habitabilité

Keywords : pollution, surveillance and counter-surveillance, citizen and participative sciences, bioindication, multispecies arrangements, habitability

\section{AUTEURS}

\section{CHRISTELLE GRAMAGLIA}

Christelle Gramaglia est sociologue au sein de l'UMR G-EAU, à l'IRSTEA de Montpellier. Ses thèmes de recherche sont 1 . La production des savoirs sur la qualité environnementale et les pollutions en situation de controverse ; 2 . Les effets sociaux des pollutions sur l'habitabilité des territoires et diverses initiatives développées pour parer aux dégradations environnementales. Elle a en charge le volet SHS du projet interdisciplinaire INTERREG SUDOE SOLPRECAIRE (2016-2019) qui vise à comparer les vulnérabilités des populations résidant dans des sites pollués Sud Européen. Elle est également chargée de cours à l'Université de Montpellier et à PontsParisTech.

\section{CHARLES-ENZO DAUPHIN}

Charles-Enzo Dauphin est titulaire d'un diplôme d'ingénieur en écologie et biosciences de l'environnement. Il est actuellement chargé de mission bioindication et responsable de l'observatoire citoyen de l'environnement au sein de l'Institut écocitoyen pour la connaissance des pollutions de Fos-sur-Mer. 